\title{
A high serum-free fatty acid level is associated with cancer
}

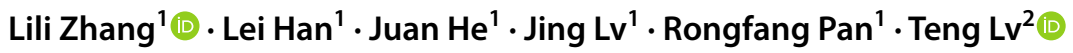

Received: 18 July 2019 / Accepted: 21 November 2019 / Published online: 26 November 2019

(c) The Author(s) 2019

\begin{abstract}
Purpose The objectives of this work were to investigate whether the serum-free fatty acid (FFA) level is meaningful in cancer patients and its role in cancer diagnosis.

Methods A total of 2206 patients were divided into a cancer group $(n=1019)$ and a noncancer group $(n=1187)$. Age, sex, body mass index (BMI), and serum FFA and serum albumin levels were collected. Cancer patients were divided into subgroups according to the location of the cancer. We then compared serum FFA levels among the tumor subgroups. A receiver operating characteristic (ROC) curve analysis was performed to further evaluate the diagnostic ability of the FFA level. SPSS 22.0 software was used to analyze the results.

Results The FFA level was higher in the cancer group than in the noncancer group. According to the multivariate analysis, there was also an increased risk of cancer associated with a high FFA level after adjusting for old age, female sex, and a low BMI. In the subgroup analysis, the FFA level in patients with lung cancer, gastric cancer, thyroid cancer, rectal cancer, colon cancer, and ovarian cancer was significantly higher than that in noncancer patients. The area under the effect-time curve (AUC) of FFAs in the whole cancer group was 0.58 , while the thyroid cancer, rectal cancer, and ovarian cancer subgroups had AUCs $>0.6$.

Conclusion Our study provides clinical evidence to support that fatty acid metabolism is associated with cancers and demonstrates that a high FFA level in the serum may be an indicator of cancer.
\end{abstract}

Keywords Free fatty acids $\cdot$ Cancer $\cdot$ Cancer biomarker $\cdot$ Early diagnosis $\cdot$ Malignant neoplasm

Teng Lv

lvteng2051@163.com

Lili Zhang

lilizhangqingdao@outlook.com

Lei Han

yy9226@126.com

Juan $\mathrm{He}$

hejuan70@sina.com

Jing Lv

songzitangguo@163.com

Rongfang Pan

panrongfang2008@163.com

1 Department of Nutrition, The Affiliated Hospital of Qingdao University, Qingdao 266000, China

2 Department of Gynaecology, The Affiliated Hospital of Qingdao University, 1677, Wutaishan Road, Xihaian District, Qingdao 266000, China

\section{Introduction}

It is estimated that there were 18.1 million new cases and 9.6 million cancer deaths worldwide in 2018 according to global cancer statistics (Bray et al. 2018). In 2018, the China Cancer Center released the National Cancer Report, which showed that in 2014, the estimated number of new cases of cancer in China was 38.04 million (21.14 million males and 16.99 million females) (Chen et al. 2018). Cancer has become one of the most crucial problems threatening human health. Cancer stage is directly related to mortality (Edwards et al. 2014; Howlader et al. 2014; Dianatinasab et al. 2018). Presymptomatic screening is related to early stage diagnosis and improved outcomes (Plumb et al. 2016; Inari et al. 2017). By increasing the cancer screening rates in those who are most likely to develop cancer, we can provide them with a definite opportunity to reduce mortality (Stich and Berlan 2004). However, most cancer patients are diagnosed at an advanced stage due to a lack of symptoms and limitations in currently available tumor markers. Free fatty acids (FFAs) 
are intermediate products of lipid mobilization that result principally from lipolysis and the breakdown of triglycerides (TGs) (Stich and Berlan 2004). Previous studies have reported that FFA levels are associated with unfavorable functional outcomes in stroke and cardiovascular disease and may play a role in the process of disease progression (Choi et al. 2014; Sun et al. 2015; Xiong et al. 2015; Duan et al. 2017; Han et al. 2018). In clinical work, we found that in some cancer patients, the FFA level was elevated even when TGs and total cholesterol (TCHO) were normal, and a high FFA level may be the only abnormality in biochemical tests. To identify whether there are differences in the FFA level between cancer and noncancer patients and the role of FFAs in the diagnosis of cancer, we designed the study described herein. If the FFA level is an indicator of cancer, patients with a high FFA level may benefit from increasing the cancer screening rates and follow-up tests.

\section{Materials and methods}

\section{Patients}

From January 2019 to June 2019, patients aged 18-65 years with normal blood lipids (serum TGs $0.3-1.92 \mathrm{mmol} / \mathrm{L}$, TCHO 2.32-5.62 mmol/L, and low-density lipoprotein cholesterol 1.9-3.12 $\mathrm{mmol} / \mathrm{L}$ ), normal liver, and kidney function (alanine aminotransferase 7-40 U/L, aspartate aminotransferase 13-35 U/L, urea nitrogen 3.1-8.8 $\mathrm{mmol} / \mathrm{L}$, and creatinine $31-132 \mu \mathrm{mol} / \mathrm{L}$ ) were extracted from the electronic medical record system of the Affiliated Hospital of Qingdao University. Patients with diabetes mellitus, hyperlipidemia, abnormal thyroid function, stroke, or cardiovascular disease and those who were pregnant were excluded. We collected body weight and height data, and the BMI (body mass index) was calculated. Depending on the diagnosis, the patients were divided into a cancer group and a noncancer group. The cancer group had definite pathological or imaging evidence, while the noncancer group did not. Tumors that could not be identified as benign or malignant were not included in any group. The general data of the two groups are shown in Table 1.

\section{Laboratory testing}

The GPO-PAP method was adopted to determine the TG level. The CHOD-POD method was adopted to determine the TCHO level. The enzyme endpoint method was applied to measure the FFA level. The FFAs that we measured were nonesterified fatty acids. Serum albumin was detected by spectrophotometry with bromocresol green. We collected only the first FFA and albumin measurements after admission. The biochemical analyzer used was Beckman Coulter AU5800 (USA).

\section{Statistical analysis}

We used the one-sample Kolmogorov-Smirnov test to examine the normality of continuous variables. The concentrations of serum FFA and albumin were naturally logarithmically transformed to improve normalization. The results are presented as percentages for categorical variables and as the mean value \pm SD or as the median value (25th percentile, 75 th percentile) for continuous variables. Differences in continuous variables were compared using Student's $t$ test (normal distribution) or the Mann-Whitney $U$ test (skewed distribution), and the distributions of categorical variables were compared using the Chi-square test. Correlations among continuous variables were assessed by the Spearman rank correlation coefficient.

The odds ratios (ORs) and 95\% confidence intervals (CIs) of the serum FFA concentration between cancer and noncancer patients were evaluated by univariate or multivariate logistic regression analyses after adjusting for age, sex, BMI, and albumin concentration. A receiver operating characteristic (ROC) curve analysis was performed to

Table 1 Patient characteristics

\begin{tabular}{llll}
\hline Characteristic & No. ofcancer patients $(n=1019)$ & $\begin{array}{l}\text { No. of noncancer } \\
\text { patients }(n=1187)\end{array}$ & $t$ or $\chi^{2}(P$ value $)$ \\
\hline Sex & & & \\
$\quad$ Male $[n(\%)]$ & $438(43 \%)$ & $730(62 \%)$ & $<0.001$ \\
Female $[n(\%)]$ & $581(57 \%)$ & $457(38 \%)$ & $<0.001$ \\
Average age (years) & $52.62 \pm 9.11$ & $47.85 \pm 13.20$ & $<0.001$ \\
BMI $\left(\mathrm{kg} / \mathrm{m}^{2}\right)$ & $24.29 \pm 4.89$ & $24.94 \pm 3.62$ & $<0.001$ \\
Laboratory findings & & & $<0.001$ \\
FFA $(\mathrm{mmol} / \mathrm{L})$ & $0.45(0.33-0.59)$ & $0.41(0.29-0.56)$ & $<0.001$ \\
Albumin $(\mathrm{mmol} / \mathrm{L})$ & $42.41(39.78-44.94)$ & $40.56(36.04-44.60)$ & \\
\hline
\end{tabular}

Average age and BMI are presented as the mean value \pm standard deviation (SD), and serum FFA and albumin levels are presented as the median value ( 25 th percentile, 75 th percentile) 
further evaluate the diagnostic ability of the FFA level. All statistical analyses were performed with SPSS software (version 22.0, Chicago, IL, USA). Statistical significance was accepted at the $P \leq 0.05$ level. AUC values of $\leq 0.5,0.5$ to $<0.7,0.7$ to $<0.8$, and 0.8 to $<0.9$ and $\geq 0.9$ indicate no, poor, acceptable, excellent and outstanding discrimination, respectively.

\section{Results}

In our study, serum FFAs were collected from 2206 patients. Overall, 1168 patients (53\%) were male and had a median age of 50 years. Patients were divided into two groups: a cancer group and a noncancer group. The main baseline characteristics of the patients are summarized in Table 1 . The cancer patients were older than the noncancer patients(52.62 vs 47.85 years, $P<0.001$ ), and the proportion of males in cancer patients was lower than that in noncancer patients ( $43 \%$ vs $62 \%$ male, $P<0.001$ ). The BMI in the cancer group was $24.29 \mathrm{~kg} / \mathrm{m}^{2}$, which was lower than that in the noncancer group $\left(24.94 \mathrm{~kg} / \mathrm{m}^{2}, P<0.001\right)$. Compared with the noncancer group, the cancer group had a higher FFA level $(0.45 \mathrm{vs} 0.41 \mathrm{mmol} / \mathrm{L}, P<0.001)$ and a higher albumin level (42.41 vs $40.56 \mathrm{mmol} / \mathrm{L}, P<0.001$ ).

As shown in Table 2, the univariate analysis revealed that old age, female sex, a low BMI, and high FFA and albumin levels were associated with an increased risk of cancer. After adjusting for age, sex, BMI, and albumin, each unit of the ln-transformed FFA values generated a $35.8 \%$ risk of cancer [OR (95\% CI): $1.358(1.126,1.638), P<0.001]$.

We divided the cancer patients into subgroups according to the location of the cancer. We then divided the patients into subgroups ( $n \geq 20$ per subgroup). Table 3 shows that patients with thyroid cancer have higher FFA levels, while patients with breast cancer have lower FFA levels. Table 4 shows that there was a significant positive correlation between FFA and BMI in noncancer and breast cancer patients. For each additional unit of BMI, the FFA level increases by $1 \%$ in noncancer patients and by $3.4 \%$ in breast cancer patients.

The Mann-Whitney $U$ test indicated that the FFA levels in patients with lung cancer, gastric cancer, thyroid cancer, rectal cancer, colon cancer, and ovarian cancer were significantly higher than those in noncancer patients (Table 5) $(P<0.05)$.

Table 6 shows that the ROC curve yielded an AUC of 0.561 (95\% CI 0.52-0.56) in the whole cancer group, demonstrating that the FFA level had poor discriminative ability to distinguish cancer patients from noncancer patients. In the subgroup analysis, all of the cancer subgroups yielded AUCs of $>0.5$, indicating that the FFA level had poor diagnostic value in lung cancer, breast cancer, gastric cancer, thyroid cancer, rectal cancer, colon cancer, esophageal cancer,
Table 2 Univariate and multivariate logistic regression analyses between groups

\begin{tabular}{llllllll}
\hline Parameter & \multicolumn{2}{l}{ Univariate analysis } & & \multicolumn{4}{l}{ Multivariate analysis } \\
\cline { 2 - 3 } & OR & $95 \% \mathrm{CI}$ & $P$ value & & OR & $95 \%$ CI & $P$ value \\
\hline Age & 1.037 & $1.029-1.045$ & $<0.001$ & & 1.065 & $1.055-1.075$ & $<0.001$ \\
Female & 2.199 & $1.787-2.531$ & $<0.001$ & & 2.237 & $1.859-2.692$ & $<0.001$ \\
BMI & 0.954 & $0.931-0.978$ & $<0.001$ & & 0.922 & $0.896-0.948$ & $<0.001$ \\
FFA & 1.56 & $1.315-1.850$ & $<0.001$ & & 1.358 & $1.126-1.638$ & $<0.001$ \\
Albumin & 1.076 & $1.059-1.094$ & $<0.001$ & & 1.131 & $1.109-1.154$ & $<0.001$ \\
\hline
\end{tabular}

FFA and albumin levels were ln-transformed in the models

Table 3 Comparison of serum FFA levels among tumor subgroups

\begin{tabular}{lcllrrrr}
\hline Group & $n$ & FFA $(\mathrm{mmol} / \mathrm{L})$ & Other cancers & $P$ & \multicolumn{1}{l}{ BMI } & Other cancers \\
\hline Lung cancer & 288 & $0.44(0.32-0.59)$ & $0.46(0.34-0.60)$ & 0.258 & $24.81 \pm 7.56$ & $24.08 \pm 3.29$ & 0.035 \\
Breast cancer & 197 & $0.42(0.27-0.55)$ & $0.48(0.36-0.63)$ & $<0.001$ & $24.49 \pm 3.20$ & $24.30 \pm 3.12$ & 0.497 \\
Gastric cancer & 146 & $0.46(0.35-0.60)$ & $0.45(0.33-0.59)$ & 0.493 & $23.26 \pm 3.21$ & $24.46 \pm 5.10$ & 0.006 \\
Thyroid cancer & 106 & $0.50(0.38-0.60)$ & $0.45(0.32-0.59)$ & 0.039 & $24.58 \pm 3.34$ & $24.25 \pm 5.05$ & 0.521 \\
Rectal cancer & 72 & $0.50(0.37-0.64)$ & $0.45(0.33-0.59)$ & 0.117 & $23.72 \pm 2.68$ & $24.33 \pm 5.02$ & 0.315 \\
Colon cancer & 66 & $0.48(0.35-0.61)$ & $0.45(0.33-0.59)$ & 0.246 & $24.59 \pm 3.43$ & $24.27 \pm 4.98$ & 0.609 \\
Esophageal cancer & 46 & $0.41(0.30-0.58)$ & $0.45(0.33-0.59)$ & 0.299 & $21.90 \pm 3.05$ & $24.40 \pm 4.94$ & $<0.001$ \\
Cervical cancer & 40 & $0.47(0.38-0.60)$ & $0.46(0.33-0.60)$ & 0.413 & $24.28 \pm 2.55$ & $24.37 \pm 3.19$ & 0.861 \\
Ovarian cancer & 20 & $0.50(0.41-0.60)$ & $0.45(0.33-0.60)$ & 0.144 & $25.30 \pm 3.24$ & $24.33 \pm 3.14$ & 0.174 \\
\hline
\end{tabular}

The BMI and FFA levels were compared among females with breast cancer, cervical cancer, ovarian cancer and other cancers 
Table 4 Relationship between FFA and BMI in the univariate regression model

\begin{tabular}{lrcl}
\hline Group & \multicolumn{1}{c}{$n$} & $\beta(95 \% \mathrm{CI})$ & $P$ \\
\hline Noncancer & 1187 & $0.010(0.002,0.018)$ & 0.020 \\
Lung cancer & 288 & $0.004(-0.004,00.012)$ & 0.287 \\
Breast cancer & 197 & $0.034(0.011,0.057)$ & 0.005 \\
Gastric cancer & 146 & $-0.007(-0.030,0.016)$ & 0.550 \\
Thyroid cancer & 106 & $-0.011(-0.031,0.010)$ & 0.313 \\
Rectal cancer & 72 & $-0.002(-0.039,0.035)$ & 0.896 \\
Colon cancer & 66 & $0.026(-0.010,0.062)$ & 0.149 \\
Esophageal cancer & 46 & $-0.000(-0.046,0.045)$ & 0.990 \\
Cervical cancer & 40 & $0.031(-0.017,0.078)$ & 0.195 \\
Ovarian cancer & 20 & $-0.001(-0.042,0.040)$ & 0.959 \\
\hline
\end{tabular}

FFA levels were ln-transformed in the univariate regression model

Table 5 Comparison of FFA levels between each cancer subgroup and noncancer patients

\begin{tabular}{lrllll}
\hline & \multicolumn{1}{l}{$n$} & Q1 & Median & Q3 & $P$ \\
\hline Noncancer & 1187 & 0.29 & 0.41 & 0.56 & - \\
Lung cancer & 288 & 0.32 & 0.44 & 0.59 & 0.017 \\
Breast cancer & 197 & 0.27 & 0.42 & 0.55 & 0.351 \\
Gastric cancer & 146 & 0.35 & 0.46 & 0.60 & 0.003 \\
Thyroid cancer & 106 & 0.38 & 0.50 & 0.60 & $<0.001$ \\
Rectal cancer & 72 & 0.37 & 0.50 & 0.64 & 0.001 \\
Colon cancer & 66 & 0.35 & 0.48 & 0.61 & 0.007 \\
Esophageal cancer & 46 & 0.30 & 0.41 & 0.58 & 0.653 \\
Cervical cancer & 40 & 0.38 & 0.47 & 0.60 & 0.084 \\
Ovarian cancer & 20 & 0.41 & 0.50 & 0.61 & 0.028 \\
\hline
\end{tabular}

$Q 1$ first quartile, $Q 3$ third quartile

The FFA levels were compared among female breast cancer, cervical cancer, ovarian cancer and noncancer patients

cervical cancer, and ovarian cancer. Among them, thyroid cancer, rectal cancer, and ovarian cancer had AUCs of $>0.6$.

\section{Discussion}

In the present study, we investigated whether the FFA level was meaningful between cancer and noncancer patients and found that the FFA level was higher in cancer patients than in noncancer patients. These findings indicate that the FFA level is pathogenically involved in cancer, which was clearly demonstrated in several studies in related fields. Recent advances in proteomics and metabolomics have deepened our understanding of the role of fatty acid metabolism in determining the fate of cancer cells (Li et al. 2017; Wang et al. 2018; Madak-Erdogan et al. 2019). The TG/FFA cycle participate various metabolic, physiological, and signaling pathways in cells. Fatty acid metabolism not only supports energy production but also plays an important role in the biosynthesis pathway, which is crucial to neogenesis (Grierson et al. 1990). Our study provides strong clinical evidence that FFAs play a role in the process of cancer progression.

As a source of metabolic energy, a substrate for cell membrane structures, and a precursor to many intracellular signaling molecules (Grierson et al. 1990), FFAs may be affected in a number of pathological conditions, such as insulin resistance, type 2 diabetes, obesity, severe liver dysfunction, hyperthyroidism, and so on(Rui 2014; Tseng et al. 2015). It is also affected by dietary and insulin fluctuations (Xin et al. 2019). Elevated FFA levels can lead to insulin resistance and other metabolic disorders (Arner 2002; Boden 2003). According to the multivariate analysis, there is an increased risk of cancer associated with a high FFA level after adjusting for other confounders. The results suggested that an elevated FFA level is an independent risk factor for cancer, especially lung cancer, gastric cancer, thyroid cancer, rectal cancer, colon cancer, and ovarian cancer. The results also showed that there was a significant positive correlation between the FFA level and $\mathrm{BMI}$ in noncancer patients and breast cancer patients,
Table 6 AUC values, cutoff values, sensitivity, and specificity of FFA levels between cancer subgroups and noncancer patients

\begin{tabular}{lllll}
\hline Subgroup & AUC $(95 \%$ CI $)$ & Sensitivity $(\%)$ & Specificity (\%) & Cut-off \\
\hline Whole cancer & 0.561 & 0.713 & 0.390 & 0.355 \\
Lung cancer & 0.545 & 0.590 & 0.500 & 0.405 \\
Breast cancer & 0.523 & 0.843 & 0.232 & 0.605 \\
Gastric cancer & 0.576 & 0.753 & 0.370 & 0.345 \\
Thyroid cancer & 0.620 & 0.670 & 0.563 & 0.435 \\
Rectal cancer & 0.614 & 0.556 & 0.648 & 0.485 \\
Colon cancer & 0.599 & 0.439 & 0.731 & 0.545 \\
Esophageal cancer & 0.520 & 0.130 & 0.939 & 0.765 \\
Cervical cancer & 0.582 & 0.800 & 0.381 & 0.365 \\
Ovarian cancer & 0.645 & 0.95 & 0.435 & 0.385 \\
\hline
\end{tabular}

The AUCs were compared among female patients with breast cancer, cervical cancer, and ovarian cancer 
while in the other cancer group, there was no significant correlation. The ROC value of the whole cancer population as a diagnostic criterion was 0.56 . As a prognostic indicator, the FFA level was weak. However, thyroid cancer, rectal cancer, and ovarian cancer subgroups had AUCs of $>0.6$, which was slightly higher than the other subgroups. This finding implies that an elevated FFA level may be a potential biomarker for cancers.

Some studies (Nayan et al. 2017; Yokomichi et al. 2017; Li et al. 2019a, b) have found that statin use in cancer improves survival outcomes and increases overall survival. Our results can also explain why lipid-lowering therapy can improve clinical outcomes since cancer patients have elevated FFA levels even when serum TGs are normal.

Our results are consistent with those of Zhang et al. (2014a, b, 2016), who performed chip-based direct-infusion nanoESI Fourier transform ion cyclotron resonance mass spectrometry (CBDInanoESI-FTICR MS) to simultaneously quantitatively and qualitatively analyze multiple targeted serumunsaturated FFAs. The authors found that unsaturated FFAs could be a potential biomarker panel for the early detection of some cancers.

Our research has certain limitations. First, we did not collect enough FFA data from healthy people. We collected data from patients who underwent the FFA test in the medical record system, and there may be certain deviations. Second, to avoid possible interference factors, we excluded patients with diabetes, hepatic insufficiency, renal insufficiency, thyroid diseases, coronary atherosclerotic heart disease, stroke, and pregnancy which may have affected the results. Since FFA levels might increase postoperatively, we collected only the first FFA measurement after admission, since it was obtained prior to surgery and had the fewest influencing factors. However, most of the tumor patients underwent inpatient surgery and were thus generally in a good condition to tolerate surgery, while the nontumor patients were inpatient general internal medicine patients who required hospitalization and were thus often in a poor condition. This may be one reason why surgical patients have higher levels of albumin than internal medicine patients. Our data on tumor distribution do not represent the incidence of tumors in our region. On the one hand, we selected cases with specified conditions. On the other hand, patients with different diseases have a preferential choice of hospital.

\section{Conclusions}

In summary, high FFA levels in the serum may be an indicator of cancer, especially when they are raised because of an unknown reason.
Author contributions JL and RP collected the data and wrote the paper, $\mathrm{JH}$ and $\mathrm{LH}$ generated the figures and wrote the paper. $\mathrm{LZ}$ performed the statistical analysis and wrote the paper. TL designed the study and wrote the paper.

Funding The authors did not receive any funding for this study.

Data availability All data generated or analyzed during this study are included in this published article and its Online Resources.

\section{Compliance with ethical standards}

Conflict of interest The authors declare no conflicts of interest to disclose.

Ethical approval This article does not contain any studies with human participants performed by any of the authors. It has been approved by the Medical Ethics Committee of the Affiliated Hospital of Qingdao University.

Open Access This article is distributed under the terms of the Creative Commons Attribution 4.0 International License (http://creativecommons.org/licenses/by/4.0/), which permits unrestricted use, distribution, and reproduction in any medium, provided you give appropriate credit to the original author(s) and the source, provide a link to the Creative Commons license, and indicate if changes were made.

\section{References}

Arner P (2002) Insulin resistance in type 2 diabetes: role of fatty acids. Diabet Metab Res Rev 18(Suppl 2):S5-S9. https://doi. org/10.1002/dmrr.254

Boden G (2003) Effects of free fatty acids (FFA) on glucose metabolism: significance for insulin resistance and type 2 diabetes. Exp Clin Endocrinol Diabetes 111:121-124. https://doi. org/10.1055/s-2003-39781

Bray F, Ferlay J, Soerjomataram I, Siegel RL, Torre LA, Jemal A (2018) Global cancer statistics 2018: GLOBOCAN estimates of incidence and mortality worldwide for 36 cancers in 185 countries. CA Cancer J Clin 68:394-424. https://doi.org/10.3322/ caac. 21492

Chen W, Sun K, Zheng R, Zeng H, Zhang S, Xia C, Yang Z, Li H, Zou X, He J (2018) Cancer incidence and mortality in China, 2014. Chin J Cancer Res 30:1-12. https://doi.org/10.21147/j.i ssn.1000-9604.2018.01.01

Choi JY, Kim JS, Kim JH, Oh K, Koh SB, Seo WK (2014) High free fatty acid level is associated with recurrent stroke in cardioembolic stroke patients. Neurology 82:1142-1148. https://doi. org/10.1212/wnl.0000000000000264

Dianatinasab M, Mohammadianpanah M, Daneshi N, Zare-Bandamiri M, Rezaeianzadeh A, Fararouei M (2018) Socioeconomic factors, health behavior, and late-stage diagnosis of breast cancer: considering the impact of delay in diagnosis. Clin Breast Cancer 18:239-245. https://doi.org/10.1016/j.clbc.2017.09.005

Duan XX, Zhang GP, Wang XB, Yu H, Wu JL, Liu KZ, Wang L, Long $X$ (2017) Elevated serum and cerebrospinal fluid free fatty acid levels are associated with unfavorable functional outcome in subjects with acute ischemic stroke. Mol Neurobiol 54:1677-1683. https://doi.org/10.1007/s12035-016-9756-y 
Edwards BK, Noone AM, Mariotto AB, Simard EP, Boscoe FP, Henley SJ, Jemal A, Cho H, Anderson RN, Kohler BA, Eheman CR, Ward EM (2014) Annual report to the nation on the status of cancer, 1975-2010, featuring prevalence of comorbidity and impact on survival among persons with lung, colorectal, breast, or prostate cancer. Cancer 120:1290-1314. https://doi.org/10.1002/ cncr.28509

Grierson HL, Wooldridge TN, Purtilo DT, Pierson J, Bast M, Wooldridge L, Armitage JO, Weisenburger DD (1990) Low proliferative activity is associated with a favorable prognosis in peripheral T-cell lymphoma. Cancer Res 50:4845-4848

Han L, Liu J, Zhu L, Tan F, Qin Y, Huang H, Yu Y (2018) Free fatty acid can induce cardiac dysfunction and alter insulin signaling pathways in the heart. Lipids Health Dis 17:185. https://doi. org/10.1186/s12944-018-0834-1

Howlader N, Mariotto AB, Woloshin S, Schwartz LM (2014) Providing clinicians and patients with actual prognosis: cancer in the context of competing causes of death. J Natl Cancer Inst Monogr 2014:255-264. https://doi.org/10.1093/jncimonographs/lgu022

Inari H, Shimizu S, Suganuma N, Yoshida T, Nakayama H, Yamanaka T, Yamanaka A, Rino Y, Masuda M (2017) A comparison of clinicopathological characteristics and long-term survival outcomes between symptomatic and screen-detected breast cancer in Japanese women. Breast Cancer 24:98-103. https://doi.org/10.1007/ s12282-016-0678-3

Li J, Condello S, Thomes-Pepin J, Ma X, Xia Y, Hurley TD, Matei D, Cheng JX (2017) Lipid desaturation is a metabolic marker andtherapeutic target of ovarian cancer stem cells. Cell Stem Cell 20:303-314.e305. https://doi.org/10.1016/j.stem.2016.11.004

Li Y, He X, Ding Y, Chen H, Sun L (2019a) Statin uses and mortality in colorectal cancer patients: an updated systematic review and meta-analysis. Cancer Med 8:3305-3313. https://doi.org/10.1002/ cam4.2151

Li YR, Ro V, Steel L, Carrigan E, Nguyen J, Williams A, So A, Tchou J (2019b) Impact of long-term lipid-lowering therapy on clinical outcomes in breast cancer. Breast Cancer Res Treat 176:669-677. https://doi.org/10.1007/s10549-019-05267-Z

Madak-Erdogan Z, Band S, Zhao YC et al (2019) Free fatty acids rewire cancer metabolism in obesity-associated breast cancer via estrogen receptor and mTOR signaling. Cancer Res 79:24942510. https://doi.org/10.1158/0008-5472.can-18-2849

Nayan M, Punjani N, Juurlink DN, Finelli A, Austin PC, Kulkarni GS, Uleryk E, Hamilton RJ (2017) Statin use and kidney cancer survival outcomes: a systematic review and meta-analysis. Cancer Treat Rev 52:105-116. https://doi.org/10.1016/j.ctrv.2016.11.009

Plumb AA, Pathiraja F, Nickerson C, Wooldrage K, Burling D, Taylor SA, Atkin WS, Halligan S (2016) Appearances of screen-detected versus symptomatic colorectal cancers at CT colonography. Eur Radiol 26:4313-4322. https://doi.org/10.1007/s00330-016-4293-7

Rui L (2014) Energy metabolism in the liver. Compr Physiol 4:177197. https://doi.org/10.1002/cphy.c130024
Stich V, Berlan M (2004) Physiological regulation of NEFA availability: lipolysis pathway. Proc Nutr Soc 63:369-374. https://doi. org/10.1079/pns2004350

Sun GJ, Ding SC, Ling WY, Wang F, Yang XP (2015) Cerebrospinal fluid free fatty acid levels are associated with stroke subtypes and severity in Chinese patients with acute ischemic stroke. World Neurosurg 84:1299-1304. https://doi.org/10.1016/j. wneu.2015.06.006

Tseng FY, Chen PL, Chen YT, Chi YC, Shih SR, Wang CY, Chen CL, Yang WS (2015) Association between serum levels of adipocyte fatty acid-binding protein and free thyroxine. Medicine (Baltimore) 94:e1798. https://doi.org/10.1097/md.0000000000001798

Wang T, Fahrmann JF, Lee H et al (2018) JAK/STAT3-regulated fatty acid beta-oxidation is critical for breast cancer stem cell selfrenewal and chemoresistance. Cell Metab 27:1357. https://doi. org/10.1016/j.cmet.2018.04.018

Xin Y, Wang Y, Chi J, Zhu X, Zhao H, Zhao S, Wang Y (2019) Elevated free fatty acid level is associated with insulin-resistant state in nondiabetic Chinese people. Diabetes Metab Syndr Obes 12:139-147. https://doi.org/10.2147/dmso.s186505

Xiong Z, Xu H, Huang X, Arnlov J, Qureshi AR, Cederholm T, Sjogren P, Lindholm B, Riserus U, Carrero JJ (2015) Nonesterified fatty acids and cardiovascular mortality in elderly men with CKD. Clin J Am Soc Nephrol 10:584-591. https://doi.org/10.2215/cjn.08830 914

Yokomichi H, Nagai A, Hirata M, Tamakoshi A, Kiyohara Y, Kamatani Y, Muto K, Ninomiya T, Matsuda K, Kubo M, Nakamura Y, Yamagata Z (2017) Statin use and all-cause and cancer mortality: biobank Japan cohort. J Epidemiol 27:S84-S91. https://doi. org/10.1016/j.je.2016.12.011

Zhang Y, He C, Qiu L, Wang Y, Zhang L, Qin X, Liu Y, Zhang D, Li Z (2014a) Serum unsaturated free Fatty acids: potential biomarkers for early detection and disease progression monitoring of non-small cell lung cancer. J Cancer 5:706-714. https://doi. org/10.7150/jca.9787

Zhang Y, Qiu L, Wang Y, He C, Qin X, Liu Y, Li Z (2014b) Unsaturated free fatty acids: a potential biomarker panel for early detection of gastric cancer. Biomarkers 19:667-673. https://doi. org/10.3109/1354750x.2014.977951

Zhang Y, He C, Qiu L, Wang Y, Qin X, Liu Y, Li Z (2016) Serum unsaturated free fatty acids: a potential biomarker panel for earlystage detection of colorectal cancer. J Cancer 7:477-483. https:// doi.org/10.7150/jca.13870

Publisher's Note Springer Nature remains neutral with regard to jurisdictional claims in published maps and institutional affiliations. 